\title{
Phytosphigosine-1-phosphate increases sensitivity of EGF-dependent cell proliferation
}

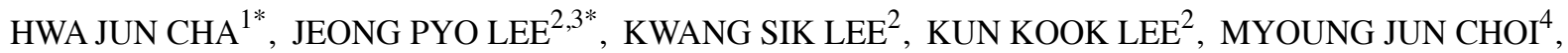 \\ DONG KYU LEE ${ }^{3}$, KWANG NYEON KIM ${ }^{5}$ and SUNGKWAN AN ${ }^{1}$ \\ ${ }^{1}$ Molecular-Targeted Drug Research Center and Korea Institute for Skin and Clinical Sciences, Konkuk University, \\ Gwangjin-gu, Seoul 143-701; ${ }^{2}$ Coreana Cosmetics Co., Ltd., Seonggeo-eup, Seobuk-gu, Cheonan-si, \\ Chungcheongnam-do 330-833; ${ }^{3}$ Industrial Engineering Chemistry, Chungbuk National University, \\ Heungdeok-gu, Cheongju, Chungcheongbuk-do 361-763; ${ }^{4}$ Phytos Co. Ltd., No. 609, \\ Industry-Academic Collaboration Foundation, Yeongtong-gu, Gyeonggi-do 443-760; \\ ${ }^{5}$ Damy Chemical Co., Ltd., No. 308, Byuk-San Digital Valley II, Keumchun-gu, Seoul 153-803, Republic of Korea
}

Received September 5, 2013; Accepted December 19, 2013

DOI: $10.3892 / \mathrm{ijmm} .2014 .1617$

\begin{abstract}
The dermis is composed of dermal fibroblasts and various synthesized extracellular matrices. Proliferation of these cells is important to skin structure homeostasis. Therefore, human dermal fibroblasts (HDFs) growth factors have been previously evaluated. In the present study, we examined whether phytosphingosine-1-phosphate $(\mathrm{PhS} 1 \mathrm{P})$ regulates gene expression, particularly cell cycle-related genes. In addition, we investigated whether there was a synergistic effect of proliferation induced by $\mathrm{PhS1P}$ and epidermal growth factor (EGF) through PhS1P-regulated genes. A microarray approach was utilized to identify gene expression changes in PhS1P-treated HDFs and data were analyzed using gene ontology (GO). In addition, proliferative synergistic effects were measured using an MTT assay. The results showed that $\mathrm{PhS1P}$ regulates various genes, particularly cell cycle-related genes. Microarray data, followed by GO, indicated that PhS1P affected the biological processes involved in the cell cycle (cyclins A2, B1 and B2). Furthermore, these genes synergistically affected EGF-dependent proliferation. The results obtained in this study demonstrated that $\mathrm{PhS1P}$ altered gene expression profiles, inducing EGF-dependent cell proliferation. Therefore, PhS1p acts as a synergistic effector for EGF.
\end{abstract}

Correspondence to: Dr Kwang Nyeon Kim, Damy Chemical Co., Ltd., No. 308, Byuk-San Digital Valley II, 481-10 Kasan-dong, Keumchun-gu, Seoul 153-803, Republic of Korea

E-mail: solideo4@gmail.com

Dr Sungkwan An, Molecular-Targeted Drug Research Center and Korea Institute for Skin and Clinical Sciences, Konkuk University, 120 Neungdong-ro, Gwangjin-gu, Seoul 143-701, Republic of Korea E-mail: ansfgrc@konkuk.ac.kr

${ }^{*}$ Contributed equally

Key words: phytosphingosine-1-phosphate, epidermal growth factor, dermal fibroblast

\section{Introduction}

Human dermal fibroblasts (HDFs) are mesenchymal cells specialized in extracellular matrix synthesis, including collagen, elastin and hyaluronic acid, in the dermis $(1,2)$. Dermal fibroblast proliferation is important in wound healing and skin structure homeostasis (3). Therefore, a growth factor was used for proliferating HDFs in cosmetics (4).

Epidermal growth factor (EGF), one of various growth factors, is a small polypeptide first purified by Cohen from the submaxillary gland of adult male mice (5). EGF is important in cell proliferation, migration and differentiation (5). These roles are mediated via activation of the EGF receptor (EGFR), a transmembrane glycoprotein with tyrosine kinase activity (6). Activated EGFR regulates proliferation and migration via activation of intrinsic signaling molecules $(7,8)$. EGF is added to serum-free media in in vitro cell culture systems as EGF is essential to cell growth (9). Furthermore, EGF exerts cytoprotective effects from cell damage, such as senescence (10). Therefore, EGF is used as an inducer of dermal fibroblast proliferation in cosmetics and medicine (4).

Phytosphingosine-1-phosphate $(\mathrm{PhS} 1 \mathrm{P})$ is derived from fungi and plants, and is structurally similar to sphingosine-1-phosphate (S1P), an endogenous signal lipid in mammalian cells $(11,12)$. PhS1P is an agonist for S1P receptors, with a particularly high affinity for $\mathrm{S}_{1} \mathrm{P}_{4}(13)$. S1P receptors include the isomers, $\mathrm{S}_{1} \mathrm{P}_{1}, \mathrm{~S}_{1} \mathrm{P}_{2}, \mathrm{~S}_{1} \mathrm{P}_{3}$ and $\mathrm{S}_{1} \mathrm{P}_{4}$, in mammalian cells (13). Since each receptor activates different downstream signals, the effects of these S1Ps are slightly different $(14,15)$. Therefore, we aimed to evaluate the PhS1P function in HDFs. The results showed that PhS1P altered gene expression and induced EGF-dependent proliferation as a synergistic effector.

\section{Materials and methods}

Cell culture and materials. HDFs were purchased from Lonza (Basel, Switzerland) and maintained in Dulbecco's modified 
Eagle's medium (DMEM; Gibco, Life Technologies, Carlsbad, CA, USA) supplemented with $10 \%$ fetal bovine serum (Sigma-Aldrich, St. Louis, MO, USA). The cells were incubated at $37^{\circ} \mathrm{C}$ in a humidified incubator containing $5 \% \mathrm{CO}_{2}$. $\mathrm{PhS1P}$ was obtained from Phytos Co., Ltd. (Suwon, Korea).

RNA extraction and microarray. Total RNA was isolated using RiboEX (GeneAll, Seoul, Korea) and quantified based on the optical density ratio $(280 / 260 \mathrm{~nm})$ using a Bioanalyzer 2100 (28S RNA/18S RNA ratio; Agilent Technologies, Santa Clara, CA, USA). Equal amounts of RNA were used to synthesize cDNA and label it with biotin using an RNA amplification kit (Ambion, Austin, TX, USA). After labeling, the microarray was hybridized with biotin-labeled RNA and streptavidin-Cy3 (Invitrogen Life Technologies, Carlsbad, CA, USA). Following hybridization, the microarray was washed using wash E1BC buffer and scanned using the iScan system (both from Illumina, Hayward, CA, USA).

Microarray analyses. Microarray data were analyzed using Genespring GX software version 11 (Agilent Technologies). mRNAs flagged 'present' in at least one sample were analyzed using fold-change. The threshold cut-off was 1.3-fold for foldchange between non-treated HDFs and PhS1P-treated HDFs. Significantly altered mRNAs were sorted using the gene ontology (GO) tool.

Quantitative polymerase chain reaction ( $q P C R)$. cDNA was synthesized using MMLV-reverse transcriptase (Invitrogen Life Technologies) according to the manufacturer's instructions. Synthesized cDNA was used for qPCR (Line gene K; Bioer Technology, Co., Ltd., China) using specific primers for cyclin A1, B1 and B2. Primers were designed by primer 3 (http://frodo.wi.mit.edu) (Table I). Expression was normalized to $\beta$-actin.

MTT assay. Cell viability was assessed using 3-(4,5-dimethylthiazol-2-yl)-2,5-diphenyltetrazolium bromide (MTT) assay. HDFs were cultured for $24 \mathrm{~h}$ in 96-well plates with PhS1P and EGF. MTT tetrazolium salt $(0.5 \mathrm{mg} / \mathrm{ml}$; Sigma) was added to cells for $4 \mathrm{~h}$. After incubation, the medium was replaced with dimethyl sulfoxide in each well. The absorbance of each sample was measured at $595 \mathrm{~nm}$ using a plate reader (Bio-Rad Laboratories, Hercules, CA, USA).

\section{Results}

PhS1P cytotoxicity in HDFs. To assess the effect of $\mathrm{PhS1P}$ on cell viability, HDFs were treated with various concentrations of PhS1P $(0,0.25,0.5$ and $1 \mu \mathrm{M})$ for $24 \mathrm{~h}$ (Fig. 1). We determined that $\mathrm{PhS} 1 \mathrm{P}$ at concentrations of $\leq 1 \mu \mathrm{M}$ had no cytotoxicity.

PhS1P alters the mRNA expression profile. To identify genes that may play a $\mathrm{PhS1P}$-dependent role, we compared the expression profiles of non-treated and $1 \mu \mathrm{M}$ PhS1Ptreated HDFs using the Illumina bead chip HumanHT-12. In 47,207 whole genes, we first filtered 35,973 genes that were flagged 'present' with a frequency higher than the sensitivity of detection in a minimum of one array. Fold-change was
Table I. Quantitative PCR primer sequences.

\begin{tabular}{|c|c|}
\hline Gene & Primer sequences \\
\hline $\mathrm{A} 2$ & $\begin{array}{l}\text { Forward 5'-TTATTGCTGGAGCTGCCTTT-3' } \\
\text { Reverse } 5 \text { '-CTCTGGTGGGTTGAGGAGAG-3' }\end{array}$ \\
\hline Cyclin B1 & $\begin{array}{l}\text { Forward 5'-CGGGAAGTCACTGGAAACAT-3' } \\
\text { Reverse 5'-AAACATGGCAGTGACACCAA-3' }\end{array}$ \\
\hline yclin B2 & $\begin{array}{l}\text { Forward 5'-TTGCAGTCCATAAACCCACA-3' } \\
\text { Reverse } 5 \text { '-GAAGCCAAGAGCAGAGCAGT-3' }\end{array}$ \\
\hline
\end{tabular}

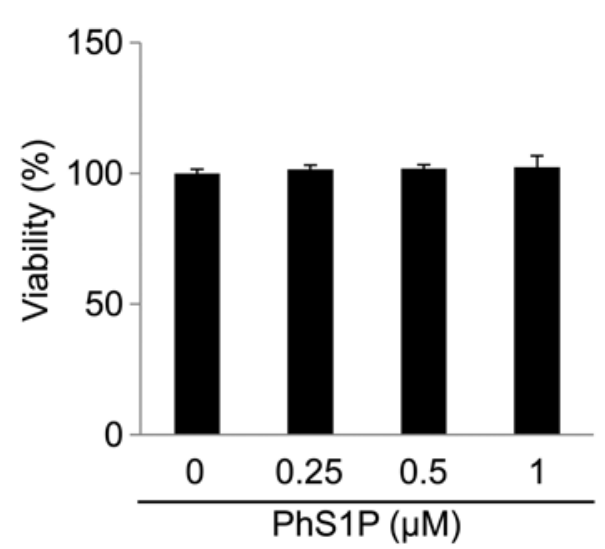

Figure 1. Phytosphingosine-1-phosphate (PhS1P) affects human dermal fibroblasts (HDFs) viability. Dose-dependent effect of PhS1P on HDF viability. HDFs $\left(5 \times 10^{4}\right.$ cells/well) were plated in 24-well plates and cultured for $24 \mathrm{~h}$ in serum-free DMEM with the indicated concentrations of PhS1P and epidermal growth factor (EGF). Cell viability was determined using MTT assay and absorbance was measured at $490 \mathrm{~nm}$. Mean values were calculated from three independent experiments. Results are expressed as the mean \pm SD.

then analyzed in these flag-filtered genes. Genes that changed by 1.3 -fold between non-treated and PhS1P-treated HDFs were presented on the heat map (Fig. 2A). The analyses identified 193 upregulated genes and 495 downregulated genes (Fig. 2B).

Using the GO analytical tool, the genes were sorted based on their roles in biological processes (Fig. 3). Genes upregulated in PhS1P-treated HDFs appeared to be involved in cell processes such as cell cycle, cell division, microtubule-based processes, chromosome segregation, cell communication, cellular responses to stimuli, and cellular component organization or biogenesis. In particular, cell cycle-related ontology (cell cycle, cell division and chromosome segregation) was significantly enriched by $\mathrm{PhS1P}$.

Cyclins A2, B1 and B2 were regulated by PhS1P. Cyclins are well-known regulators in cells (16). Therefore, the analyses revealed that $\mathrm{PhS1P}$ affected cell proliferation by altering specific mRNAs. In addition, we confirmed the mRNA expression change of cyclin A2, B1 and B2 using qPCR (Fig. 4).

We also examined the synergistic effect of PhS1P and EGF on HDF viability. Compared with PhS1P-treated HDFs in the absence of EGF, the viability of PhS1P-treated HDFs with EGF was markedly increased (Fig. 4D). These data indicate that $\mathrm{PhS1P}$ is a co-effector in the induction of EGF-dependent cell proliferation. 


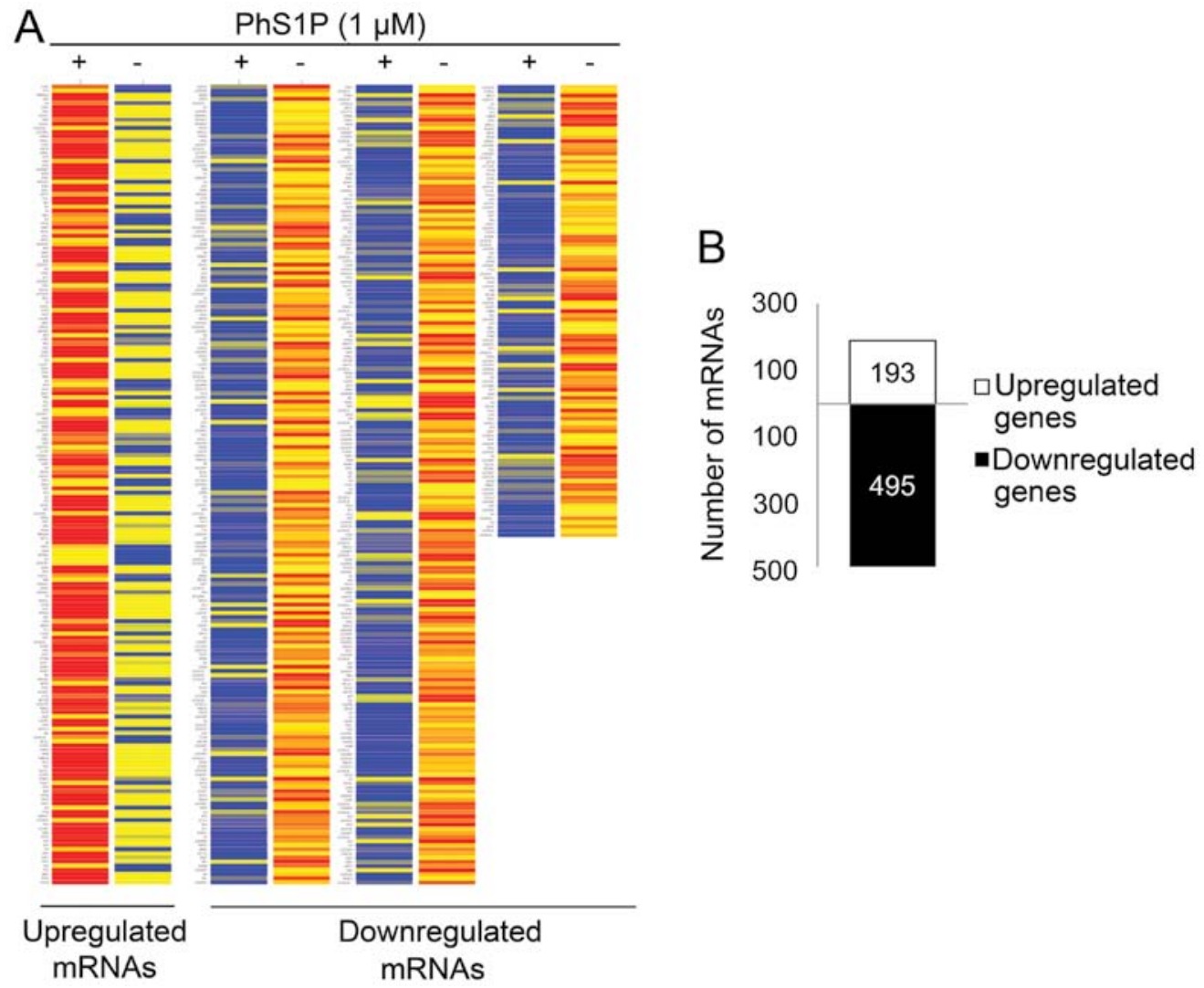

Figure 2. Phytosphingosine-1-phosphate (PhS1P) regulates the gene expression profile in human dermal fibroblasts (HDFs). HDFs were treated with $\mathrm{PhS1P}(1 \mu \mathrm{M})$ for $24 \mathrm{~h}$, and total RNA was extracted as described in Materials and methods. Changes in gene expression were determined by cDNA microarray gene profiling using the Illumina Human Bead-Chip array. A fold-change of $>1.3$-fold was considered differential expression. (A) Heat map of upregulated and downregulated genes. Red and blue colors show relative expression intensity. (B) Number of mRNA altered by $1 \mu \mathrm{M}$ PhS1P in HDFs.

\section{Biological function}

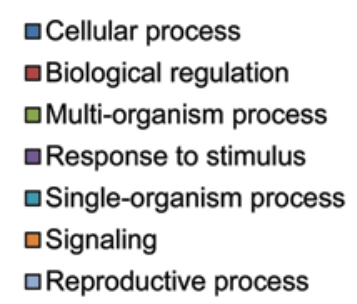

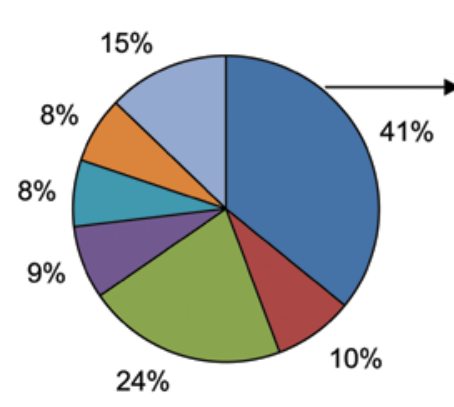

\section{Cellular component}

¿Cell part
घOrganelle part
口Organelle
$\square$ Macromolecular complex
$\square$ Cell

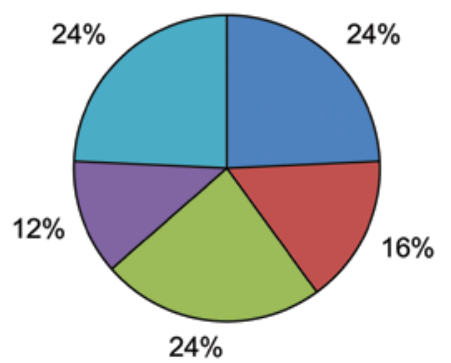

Figure 3. Gene ontology (GO) analyses of phytosphingosine-1-phosphate (PhS1P)-related genes in human dermal fibroblasts (HDFs). Enriched GO categories $(\mathrm{P}<0.01)$ were determined by counting ontology of up- and downregulated genes in biological processes. Gene classification was performed according to gene ontology terms: i) biological function, ii) cellular component and iii) molecular function. However, molecular function was not analyzed in the GO analyses. 

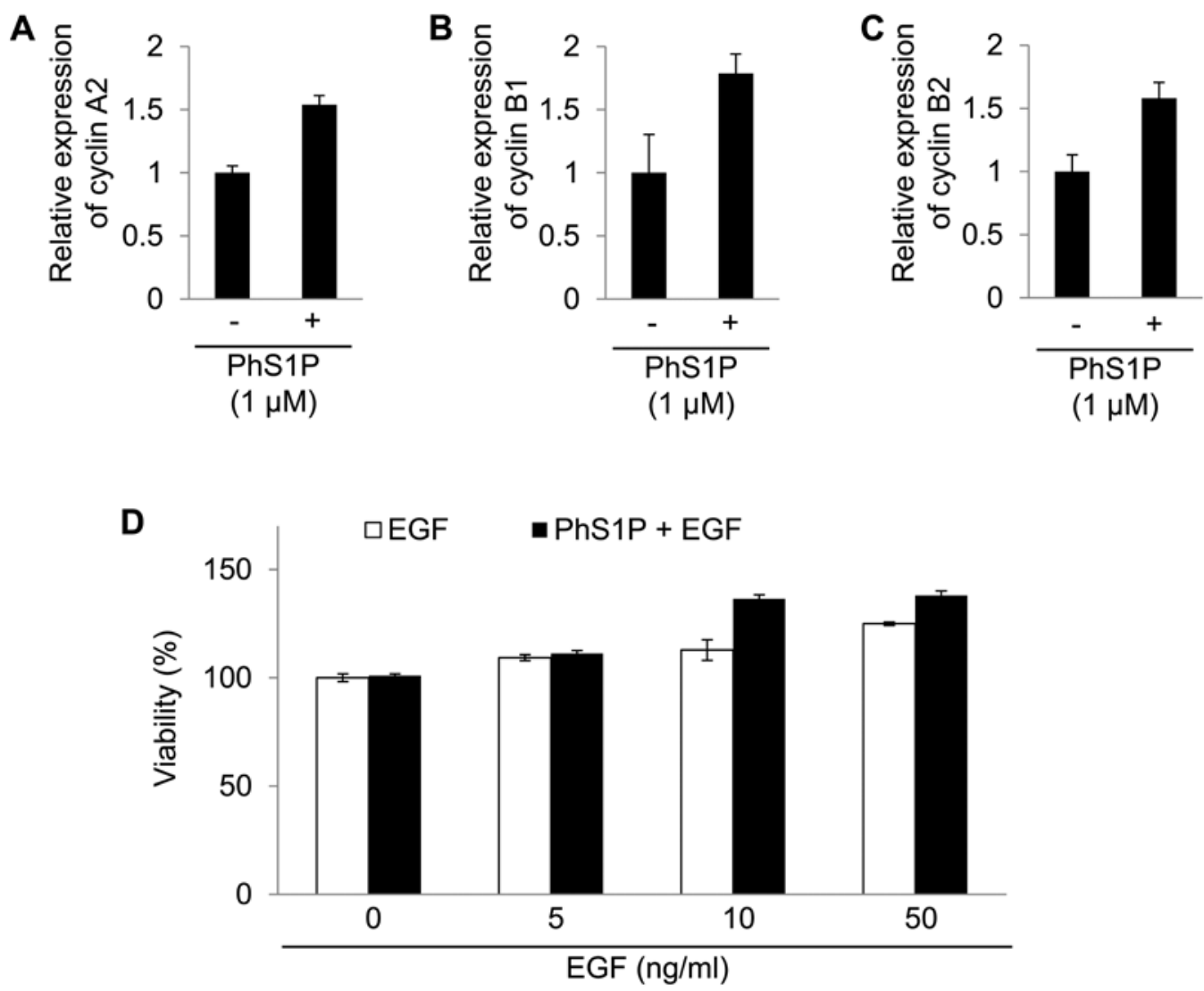

Figure 4. Phytosphingosine-1-phosphate (PhS1P) regulates cell cycle-related genes and shows the proliferative synergistic effect with epidermal growth factor (EGF) in human dermal fibroblasts (HDFs). Total RNA was isolated from PhS1P-treated and non-treated HDFs. Results are expressed as the mean \pm SD in triplicate. (A) Cyclin A2, (B) cyclin B1 and (C) cyclin B2. (D) Proliferative synergistic effect of PhS1P and EGF. HDFs (5x104 cells/well) were plated in 24-well plates and cultured for $24 \mathrm{~h}$ in serum-free DMEM with the indicated concentrations of PhS1P and EGF. Cell viability was determined using MTT assay and absorbance was measured at $490 \mathrm{~nm}$. Mean values were calculated from three independent experiments. Results are expressed as the mean $\pm \mathrm{SD}$.

\section{Discussion}

The aim of the study was to evaluate the effect of PhS1P on EGF-induced proliferation in HDFs. PhS1P is phytochemically derived from fungi, plants, and even mammalian cells (12). In a previous study, it was shown that $\mathrm{PhS1P}$ protects against hydrogen peroxide-dependent growth arrest (17). Recent data from Lee et al (17) suggested that PhS1P had no significant effect on the proliferation at concentrations $<1 \mu \mathrm{M}$ in HDFs (Fig. 1). However, PhS1P $(1 \mu \mathrm{M})$ regulates various cell cycle-related genes in HDFs (Fig. 2). In particular, the cyclins (cyclin A2, B1 and B2), master regulators of the cell cycle, were upregulated by PhS1P. Cyclin A2 regulates S-phase progression and entry into mitosis $(18,19)$. During $\mathrm{S}$ phase, cyclin A2 initiates DNA synthesis (20). During the $\mathrm{G} / \mathrm{M}$ phase, cyclin $\mathrm{A} 2$ triggers entry into mitosis by activating cyclin B1-Cdk1 (21).

In the progressing cell cycle, a sustained high expression of cyclins is essential (16). However, overexpression of cyclins is insufficient to induce cell cycle progression $(22,23)$. As shown in Fig. 2, PhS1P upregulated mRNA expression of cyclin A2, B1 and B2 although there was no change in cell viability (Figs. 1 and 2).

Combining the present data with our previous data (17), we determined that $<1 \mu \mathrm{M} \mathrm{PhS1P}$ increases cyclin expression, but does not affect viability. Moreover, EGF triggered proliferation in PhS1P-treated HDFs (Fig. 4D). Therefore, together with the results from Ikezawa et al (22), our data suggest that a cyclin-enriched condition results in synergistic growth following treatment with growth factors, such as EGF (24).

In summary, results of the present study have shown that PhS1P regulates cell cycle-related genes. In addition, the changes in gene expression synergistically trigger EGF-induced proliferation.

\section{Acknowledgements}

We would like to thank all other members of Damy Chemical Co., Ltd. for their support. This study was supported by the Konkuk University in 2012.

\section{References}

1. Crigler L, Kazhanie A, Yoon TJ, Zakhari J, Anders J, Taylor B and Virador VM: Isolation of a mesenchymal cell population from murine dermis that contains progenitors of multiple cell lineages. FASEB J 21: 2050-2063, 2007.

2. Giro MG, Oikarinen AI, Oikarinen H, Sephel G, Uitto J and Davidson JM: Demonstration of elastin gene expression in human skin fibroblast cultures and reduced tropoelastin production by cells from a patient with atrophoderma. J Clin Invest 75: 672-628, 1985. 
3. Schreier T, Degen E and Baschong W: Fibroblast migration and proliferation during in vitro wound healing. A quantitative comparison between various growth factors and a low molecular weight blood dialysate used in the clinic to normalize impaired wound healing. Res Exp Med (Berl) 193: 195-205, 1993.

4. Allen G: Cosmetics - chemical technology or biotechnology? Int J Cosmet Sci 6: 61-69, 1984.

5. Carpenter G and Cohen S: Epidermal growth factor. J Biol Chem 265: 7709-7712, 1990 .

6. Cohen S: The receptor for epidermal growth factor functions as a tyrosyl-specific kinase. Prog Nucleic Acid Res Mol Biol 29: 245-247, 1983.

7. Dittmar T, Husemann A, Schewe Y, Nofer JR, Niggemann B, Zänker KS and Brandt BH: Induction of cancer cell migration by epidermal growth factor is initiated by specific phosphorylation of tyrosine 1248 of c-erbB-2 receptor via EGFR. FASEB J 16 $1823-1825,2002$.

8. Andl CD, Mizushima T, Nakagawa H, Oyama K, Harada H, Chruma K, Herlyn M and Rustgi AK: Epidermal growth factor receptor mediates increased cell proliferation, migration, and aggregation in esophageal keratinocytes in vitro and in vivo. $\mathrm{J}$ Biol Chem 278: 1824-1830, 2003.

9. Halleux $\mathrm{C}$ and Schneider YJ: Iron absorption by $\mathrm{CaCo}_{2}$ cells cultivated in serum-free medium as in vitro model of the human intestinal epithelial barrier. J Cell Physiol 158: 17-28, 1994.

10. Shiraha H, Gupta K, Drabik K and Wells A: Aging fibroblasts present reduced epidermal growth factor (EGF) responsiveness due to preferential loss of EGF receptors. J Biol Chem 275 : 19343-19351, 2000.

11. Kim MK, Park KS, Lee H, Kim YD, Yun J and Bae YS: Phytosphingosine-1-phosphate stimulates chemotactic migration of L2071 mouse fibroblasts via pertussis toxin-sensitive G-proteins. Exp Mol Med 39: 185-194, 2007.

12. Pata MO, Hannun YA and Ng CK: Plant sphingolipids: decoding the enigma of the Sphinx. New Phytol 185: 611-630, 2010.

13. Inagaki Y, Pham TT, Fujiwara Y, Kohno T, Osborne DA, Igarashi Y, Tigyi G and Parrill AL: Sphingosine 1-phosphate analogue recognition and selectivity at S1P4 within the endothelial differentiation gene family of receptors. Biochem J 389: $187-195,2005$.
14. Takuwa N, Du W, Kaneko E, Okamoto Y, Yoshioka K and Takuwa Y: Tumor-suppressive sphingosine-1-phosphate receptor-2 counteracting tumor-promoting sphingosine-1-phosphate receptor-1 and sphingosine kinase 1 - Jekyll Hidden behind Hyde. Am J Cancer Res 1: 460-481, 2011.

15. Takuwa Y, Du W, Qi X, Okamoto Y, Takuwa N and Yoshioka K: Roles of sphingosine-1-phosphate signaling in angiogenesis. World J Biol Chem 1: 298-306, 2010.

16. Malumbres $\mathrm{M}$ and Barbacid $\mathrm{M}$ : Cell cycle, CDKs and cancer: a changing paradigm. Nat Rev Cancer 9: 153-166, 2009.

17. Lee JP, Cha HJ, Lee KS, Lee KK, Son JH, Kim KN, Lee DK and An S: Phytosphingosine-1-phosphate represses the hydrogen peroxide-induced activation of c-Jun $\mathrm{N}$-terminal kinase in human dermal fibroblasts through the phosphatidylinositol 3-kinase/Akt pathway. Arch Dermatol Res 304: 673-678, 2012.

18. Ohashi A, Imai H and Minami N: Cyclin A2 is phosphorylated during the G2/M transition in mouse two-cell embryos. Mol Reprod Dev 66: 343-348, 2003.

19. Alexandrow MG and Hamlin JL: Cdc6 chromatin affinity is unaffected by serine-54 phosphorylation, S-phase progression, and overexpression of cyclin A. Mol Cell Biol 24: 1614-1627, 2004.

20. Yam CH, Fung TK and Poon RY: Cyclin A in cell cycle control and cancer. Cell Mol Life Sci 59: 1317-1326, 2002.

21. Fung TK, Ma HT and Poon RY: Specialized roles of the two mitotic cyclins in somatic cells: cyclin A as an activator of $\mathrm{M}$ phase-promoting factor. Mol Biol Cell 18: 1861-1873, 2007.

22. Ikezawa K, Ohtsubo M, Norwood TH and Narayanan AS: Role of cyclin $\mathrm{E}$ and cyclin E-dependent kinase in mitogenic stimulation by cementum-derived growth factor in human fibroblasts. FASEB J 12: 1233-1239, 1998.

23. Chou JL, Fan Z, DeBlasio T, Koff A, Rosen N and Mendelsohn J: Constitutive overexpression of cyclin D1 in human breast epithelial cells does not prevent G1 arrest induced by deprivation of epidermal growth factor. Breast Cancer Res Treat 55: 267-283, 1999.

24. Fisher D, KrasinskaL, Coudreuse D and Novák B: Phosphorylation network dynamics in the control of cell cycle transitions. J Cell Sci 125: 4703-4711, 2012. 